\section{AEROBIC EXERCISES IN HEART FAILURE - A REVIEW}

\section{ABSTRAC}

OBJECTIVE

to determine the effect of aerobic exercise training on the quality of life QoL. morbidity, mortality of patients with Heart Failure (HF)

\section{SEARCH STRATEG}

EBSCO Host, Medine, Cochrane database, CINHAL, Ovid, AMED were the databases searched, from 1999 to 2010 which was the peak era of implementa-

\section{SELECTION CRITERIA}

The studies that involved aerobic exercise either in combination or alone were
included according to the criteria of New York Heart Association (NYHA) class IIII HF patients. Randomized control trails (RTCS) and systematic reviews were

\section{DATA COLLECTION AND ANALYSIS}

The screening of the papers was done by three review authors. The initial screensecond and third.

\section{MAIN RESULTS}

10 articles met the inclusion criteria including 3 systematic reviews. 3647 particic$30-82$ years, where moles were dominating in almost all studies. Thas from reveals no statistical differences in the mortality and morbidity. There was no individual effect of aerobic exercise on QoL whereas better results were eviewed when performed in combination.

\section{CONCLUSION}

Aerobic exercises have improved the physiological function, aerobic capacity
and quality of life among the patients suffering with HF. However, these exercise therapies were given in combination with resistance training but distinc review.

\section{INTRODUCTION}

Heart Failure (HF) has been prevalent throughout the
world as the leading cause of morbidity and mortality which increases the incidence with the growing artality has been estimated that more than 550,000 patien th suffering with Chronic Heart Failure (CHF) at least once in their lifetime 4 . Several causes may lead to $\mathrm{HF}$ but myocarcauses specifically systolic that is: there is a reduced ventricular contractions. Unfortunately its prevalence has not been investigated by the researchers in Pakistan. However, the efiology of CHF has been examined in on of thised rest during cardiac phase I and II. resulting in prolong rehabilitation?. With the advent of time, the importance of early mobilization out of bed and physical activity is highlighted and encouraged ${ }^{8}$. A number of
Activities of Daily Living (ADLS) were introduced in the cardiac rehabilitation center but remained a challenge especially in Pakistan.

nability to perform aerobic exercise was considered as of the pharmacological therapy, the prognosis of the $\mathrm{HF}$ patients is poor but exercise training has markedly show improvement in exercise capacity' ${ }^{10}$, quality of life on have been increased due to fotigue and dyspnea which may be altered by exercise training Aerobic exercise trainings have been well established than non-pharmacological treatment presenting its Qol. Moreover the mortality and morbidity of the patients with HF has also decreased.

The objective of this review is to reveal the importance of aerobic exercises among patients suffering with HF in Pakistan.

\section{METHODS}

Search \& Keywords

OSSCO Host, Medline, Cochrane database, CINHAL Ovid, AMED were the databases searched, from 1998 to exercises for $H F$ and other cardiac diseases perobic some American and a few European journals were inaccessible. The key words such as aerobic exercises, chronic heart failure, exercise training, left ventricula quality of life, mortality, morbidity, walking, treadmil cycling ergometer were used.

Study Selection \& Data Collection Proces

作iews and one meta-andysis were rew; 3 systematic the studies emphasized on aerobic exercise alone bu these exercise trainings were performed in almost all the reviewed studles ether as a single intervention or combined with resistance exercise program to assess the
mpact on the outcome measures. The studies selected used oxygen consumption capacity, morbidity, mortality and fatigue as their outcome measures. 4 of 10 studies aerobic and resistance exercises, none of the study selected used resistance training only. The effectiveness of exercise based rehabilitation in patients with CHF has been demonstaled in the earlier ystematic reviews the same yet a few differences in the outcome measures

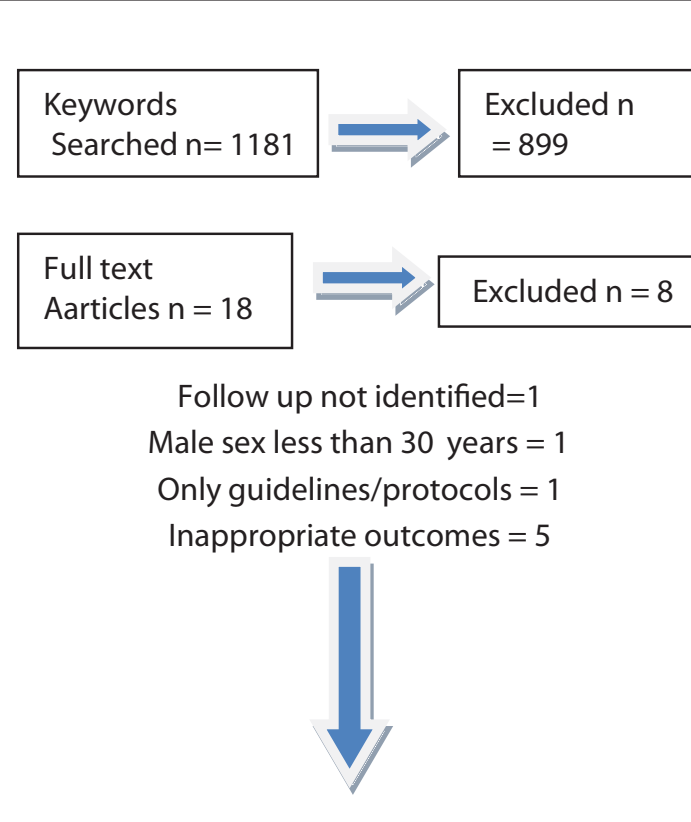

Total RCTs, systematic reviews included $=10$

Summary of study selection

were noticed. Recently, Davies et alrs selected 19 Ran omized Control Trials (RCTs) with almost 3647 patients; Quality of Life (HRQOL) mortality hospitalization and cos effectiveness was also reviewed. The maximum sample size considered in any of the 19 RCTs was 200 patients where maximum number of male patients was selected. members whereas the remaining consists of more than $72 \%$ of them. The recruited subjects were without any complications fulfiling the New York Health Association (NYHA) class II and III criteria. The mean age selected for aerobic and resistance training were used and different these studies were mostly conducted in Europe and United States of America whereas only I was conducted were based on exercises-only interventions. However the psychological intervention was also provided in one of the studies. The duration of the training programs were It was mostly an outpatient program whereas one week. study was home based. The interventional group received the cardiac care whereas the control group recelved no intervention at all, Just routine medicines. The MLWHE, whereas others used Psychological Wellbeing Index (PGWB), Kansas City Cardiomyopathy Questionnaire (KCCQ), Spritzer's Quality of Life Index (QLI) and Patients Global Assessment of Quality of Life (PGAQoL). ment with aerobic exercise whereas remaining showed a few limitations. However, while assessing the effects on mortality; no statistically significant results were obtained in the study. These results remain similar with the previous the exercise and the control group. 
Rees et allo by performing RCTs select all the adults with interventions compared with the usual medical care on the mortality, morbidity, exercise capacity and QoL. Al ed. Data was extracted from 29 studies which met the
inclusion criteria. 24 studies measured the maximum oxygen capacity (VO2 max), 15 studies measured the exercise duration and 6 studies recorded the work ejection fraction of less than $40 \%$. The mean ages ranged from $51-77$ years. All the studies selected for the review
dominated the male patients and may be considered as dominated the male patients and may be considered as
one of the major limitations' ${ }^{6}$; only 2 studies recruited the female patients. The VO2 max measured in 24 studies with 848 patients randomly allocated. Large improvemen effects were seen in $\mathrm{VO} 2 \mathrm{max}$ with the exercise training grous by $2.16 \mathrm{~m} / \mathrm{kg} / \mathrm{min}$. 23 out of 29 trials evaluated an resistance training of the peripheral muscle group. It may be considered that aerobic training may have contributed more to increase the exercise capacity. A sensitivity analysis was carried out by excluding the studies tha
focused completely on the resistance training in determine the impact of aerobic exercise. Results showed that it had no significant effects on the pooled analysis for the outcome of $\mathrm{VO} 2$ max. The exercise increment. Greater improvements were seen in patien aged less than 55 years since age is reported as an individual data. The duration was measured in 15 studies work capacity by 15.1 watts It may be assumed that the increasing follow up may have increased the effect of the outcome measures. Exercise capacity was assessed Walk Test (6MWT) and the physical work on 6 Minute Walk Test (6MWT) and the physical work capacity. The
distance on 6MWT was measured in 282 patients; that increased by $40.9 \mathrm{~m}$. It is significant to improve the exercise capacity as decrease in it, restricts the ADL

Rees et allo also reported the results that were obtained
from the 9 trials out of 29 on HRQoL as an outcome meas from the 9 trials out of 29 on HRQoL as an outcome measure. Different intervention groups (aerobic \& resistance)
uses different scales in order to measure the HRQOL. 7 out of 9 studies showed improvements in the intervention
group then in the control group 5 studies used the grisease specific Minnesota Living with Health Failure
ging Questionnairel 7 where significant improvements were the HRQoL for a longer duration and 1 of these showed sustained improvement which was maintained over an extended period of time. Aerobic exercises improved the
QoL by improving the physical activity of the patients with HF, but all aerobic capacity depends on the Freaventh Intensity, Time and Type (FIT) of exercises performed ${ }^{18}$. It may also depend on modalities used, more commonly
treadmill and bicycle ergometer?. No generalized guidetreadmill and bicycle ergometer?. No generalized guide-
lines for all heart failure were recommended precisely but
individual modifications were required.

Previously, Lloyds-Williams' also investigated the effects of exercise training on the patients with CHF, aged between
$50-82$ years. They were randomly selected to assess the physical performance (e.g. increased peak oxygen
uptake, cardiac output, aerobic capacity, mortality and QoL. The studies which have the influence of drugs on the physical performance of the patients and affecting the
outcome measure were excluded; along with it those studies consisting of the biomedical changes due to
exercise training were also excluded. All the studies reporting the languages other than English were also placed in the exclusion criteria. Wide range of databases
from 1996 to 2000 was used and relevant American and European journals were studied but due to unavailability of some electronic journals the data was scrutinized. CINHAL and AMED were not surfed to find the studies. Out of 31 studies 14 were prospective RCTs, 8 were
randomized crossover trails, 2 were non randomized control trials and 7 were pre-test and posttest studies. The total number of participants included in the study was and only 1 study focused ag females exclusive the review 31,10 studies were exclusively focusing on males. Different exercise trainings were included in the review but in most of the studies aerobic trainings were implemented. Although aerobic exercise was not clearly
mentioned in the study nor differentiated but the training modalities mentioned in the study made it clear. Mostly supervised, hospital based program was included in this review and the patients used a cycle ergometer commonly. Other modalities were also used in combina-
tions such as swimming, jogging, walking and different
circuittrainings. Activities such as step exercises, calisthenics, ball games, resistance exercises, and anaerobic training were also focused. Home based programs were
included in the review and in 7 studies patients with crcle ergometer and treadmill were given for home use. All
these programs were short termed with duration of 4 to 52 these programs were short termed with duration of 4 to 52
weeks. The frequency to perform aerobic exercises was ranged from 1 to 7 times per week; with the session lasting between 10 to 60 minutes. A descriptive synthesis was
undertaken but the difference between the studies was not investigated. The amount of oxygen uptake was not
ranged but was tabulated and most of the patients were working on mild to moderate intensity; ranged $50 \%$ to $70 \%$ R. Mostly the studies that were included were of small
sample size less than 50 participants. The patients included were mostly convenient samples and were of younger age whereas the major population age of HF is much
higher. The HF patients with comorbidities were excluded higher. The HF patients with comorbilities were excluded
so it cannot be generalized to the vast population. There was no classification of the patient mentioned according to the risk stratification or any other classification. A
number of discrepancies were present in the number of number of discrepancies were present in the number of sudies included such as a lack of precision in study
designs were found. The training programs were of small duration and mostly lasted for 8 weeks or less whereas only two studies lasted for a year. 16 studies measured the
Qot and 11 were found to be beneficial, although small duration decreased the usefulness due to short duration.

Short term exercise training was found to be beneficial in
the selected groups of patients and physiological Improvements were notified among these patients.
Excentse Exercise training has shown positive effects but it may be
difficult to evaluate whether the improvements were due to aerobic alone or in combination with resistance exercises.

CHF occurs when heart is unable to pump sufficiently eading to exertion, breathlessness and fatigues, as one of
the major symptoms. Inability to perform the aerobic exercise is the characteristic feature of heart failure' and training on fatigue and dyspnea in the HF so that the central hemodynamics and the cardiac pumping capacity can be improved. Aerobic training may contribute to decrease the exercise intolerance, fatigue and to see the effect of 24-week exercise training on fatigue and heart failure. 19 male and 2 females where recruited
by a RCCS. The randomization was done in the ratio of 2:
that is; twice the intervention group when compared with that is; twice the intervention group when compared with
the control group. The average age among them was 66 years with an ejection fraction of $28.447 .4 \%$. These patients were classified according to the NYHA in the
class II, III and IV. The eligibility criteria also included the patients who were able to read and speak English. The week program was given to these patients $<40 \%$. A 24 and resistive training at a frequency of 3 times per week was given and each session of 60 minutes was allotted. Aerobic exercises were given for 30 minutes excluding the (a) bike, rowers and arm ergometers. Piper Fatigue Scale (PFS) and Dyspnea Index (DI) were used to measured was given and the patients were advised to work $a$ th intensity of 60-80\% of VO2 max and the Rate of Perceived Exertion (RPE) should be 12-14 on the BORG Scale. 23 subjects were selected out of which only 21 completed the study but I patient from the control group died of
Myocardial Infarction. I subject was diagnosed with Mancer and was unable to continue the exercise Patient remained on their pharmacological treatment where
ACE inhibitors, B Blockers and diuretics were prescribed. Results showed marked improvements in fatigue overtime in exercise trained heart failure $(p<0.05)$ on the PFS scale where as significant decrease in sensory subscale of PFS in the training group was noticed. However, non-significan
results were obtained on the DI. The average adherence rate for the exercise group over 24 weeks intervention wa the perceived sensory fatigue of the trained subjects,
slight improvements were seen in the control group bu slight improvements were seen in the control group bu
only till 12 weeks then these were worsened by 24 weeks. A few limitations were there in the study; a lack of sample size and statistical power was the major obstace.
ore, this study may atribute to overcome the symptom experienced by HF patients but it would be difficult to confirm which intervention might have been significant.
is considered that aerobic training has decreased fati whereas resistance training may have improved the
strength of the muscle'

Similar research was conducted by Witham et alpo but the sample of convenience of 26 patients aged more than 70 years was recruited, out of which the follow-up of only 15 according to NYHA. Patients suffering from aortic stenosis sustained ventricular arrhythmia, uncontrolled atrid
fibrillation, and unstable angina were excluded from the study. Hospital Anxiety and Depression Score (HADS) Heart Failure Quality of Life Questionnaire Chronic measured. The two week program consisted of both aerobic and resisted training, of both the upper and ower limbs performed in both standing and sittin and its effectiveness was tested for the 6 MWT at baseline and after the program. A proper warm up and cool dow phases were considered. These aerobic exercises
prepared the body for the work load on the heart's. prepared the body for the work load on the heart"s.
Although timings of warm-up and cool down were no Although timings of warm-up and cool down were no
mentioned in the methodology neither a separate recommendations were given especially for the hear failure patients but in general a warm up for 15 minutes for
cardiac patients with $\mathrm{HF}$ are recommended $\mathrm{d}^{2}$. The cardiac patients with HF are recommended'2. The
exercises were easily completed by the patients whereno monitoring was undertaken although it was a group of older aged people. Although it was a pilot study so the conclusion of efficacy of interventions cannot be drawn out of it still the patients showed good toleration for the
training program. Only one adverse event was recorded volving a fall from the step so the interventions may be considered safe. The results showed an increase in state whether this increment on the 6MWT was due to aerobic or resisted exercises. Furthermore, the attend-
ance was $80 \%$ for the program emphasizing that the patients may have increased the physical activity but the provision of taxi transport may have induced an element
of bias in it. Overall, the two weekly groups focusing on of bias in it. Overall, the two weekly groups focusing on aerobic endurance and everyday functional task was
accepted and tolerated.

Several small studies have shown that aerobic exercises Chrease the exercise capacity of patients suffering with
CHF. Four different studies were conducted using aerobic exercises as their intervention protocol (Yamomto et $\mathrm{al}^{22}$,

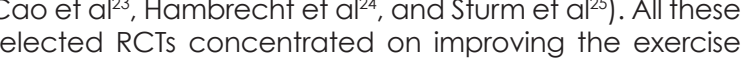
capacity of patients suffering with $\mathrm{HF}$, a sample size of 125
patients $(8,26,73,18)$ was recruited from all these studies. Only one study had a larger sample size ${ }^{25}$ the rest had a sample size less than 30 . Moreover, only one of these studies measured the effects of exercise (aerobic patients foll in the categony of NHYA in Class I and or hese remained dominant as being in the other studies. The study conducted by Hambrecht recruited 73 males only. Step aerobics, cycle, treadmill, bike, arm weight, arm
ergometer walking, calisthenics, ball games, bicycle ergometers were used as intervention modes. One study measured the effects of both aerobic and resistance

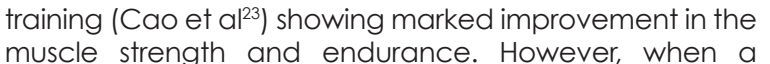
comparison was made between the upper and lower more pronounced in the lower extremity. Moreover, resistance exercised groups improved in the muscle biceps, quads and hamstrings). Aerobic doinis improved more of cardiopulmonary capacity. The combination of aerobic and resistance exercises improved the pulmonary fitness, muscle strength and
reduced the fatigue. These results were similar with other reduced
studies

A home based program was used by Hambrecht et al12 in the study where patients were randomly assigned to 2 for 10 minutes and 4 - 6 times per day, followed by a 20 minutes per day at $70 \%$ peak oxygen uptake to a no intervention in the control group. The central hemodynuring the exercise. Echocardiography was conducted to determine the left ventricular diameters and volume; and baseline follow up of 6 months was given for the trained group had statistically significant improvements when compared with the control group. Maximum ventilation, exercise capacity, and exercise time also showed marked progress. A decrease in heart rate and in the peripheral resistance occurred with small but were obtained for the remaining two studies
wally

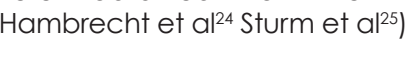

These studies conducted mostly focused on laboratory 
based or out-patient programs where patient exercise
under the controlled conditions but these exercises was not applicable for all the patients due to inaccessibility of the formal program. Many home-based programs hav been established for these patients. Dracup et al
randomized 173 with systolic heart failure. Out of which 87 were placed in the control group and 86 were allocated a home-based exercise program. The patients aged between 18 - 80 years were all English speaking people.
The NYHA II classification criteria were followed whereas the patients with myocardial infarction were all excluded from the study. The exercise groups were assigned to perform a graduared, low exerise and resistance tranisitAerobic exerise was initially given for 10 minute at a maximal heart rate of $40 \%$ and progressively an increment up to 45 minutes at $60 \%$ of HR for the remainder program. The patients were given a prescription for their minutes of walking. After 6 weeks, the aerobic training was optimized and resisted training was added. Upper and lower body strengthening exercises for 3 days pe week was given, pedometers were used which has found
to be reliable? and minutes walked on weekly and then on monthly visis. Composite end points were selected over a single
outcome to increase the number of events over 1 yed outcome to increase the number of events over 1 year hospitalizations were selected mover HE. The and all-cause probability of having coronary heart disease. Neithe deaths nor exercise related injuries were reported in the
study. The results from the study indicated that there was study. The resulls from the sudy is dicated that there wos ns for both the exercise group and contro group.

\section{SUMMARY OF EVIDENC}

Aerobic training, as part of comprehensive program has shown highly significant improvements in function $\mathrm{ty}$ in the individuals with $\mathrm{HF}$ but the effects were sho termed. It has also shown butral adaptation to the exercising patients with CHF although initially it was
thought that it is due to peripheral adaptations ${ }^{2}$. The studies suggest that moderate intensity exercise training according to the FIIT principal. Continuation of exercis training must be till the results are beneficial. Mostly the trials are hospital based and home programs are not kep advised among these patients, It has been prescribed that 30 minutes of activity daily or in 3 different bouts sufficient for a patient's. Various studies have been conducted to determine the optimum frequency for cardiac

\section{LIMITATIONS OF THE STUDY}

A large amount of literature is available concerning fraining for the patients with HF although methodologica review consist of meta-analysis and systematic review due to which most of the trials where covered. Trials often short duration that does not make it a representative of the large population of CHF. There are some marked limitations in the review. Firstly, both home based and hospital based studies are considered. Although, the outcomes remain lifferent in both the environment that
has been recently explained by Piepoli et al $2011^{8}$. There are some evidences that exercise training improves thials have looked at the mortality over a sustained
period?. All these studies have considered different regimes and varied outcome measures but generalization regarding exercise training is difficult. Although a forobic exercise has been recommended as a vital part macological approach in the management of $\mathrm{CHF}$ yet
the significance of these trainings among sleep related bnormalities has not been studies well. However, some significant results are obtained for the patients suffering
with sleep apnea ${ }^{22}$.I ti s recommended that further studies particularly among the elderly patients and those with more severe CHF should be conducted with a larger sample size. Also, the follow-up of the patients are not

\section{CONCLUSION}

Exercise training is recommended for all the patient suffering with $\mathrm{HF}$ if they fulfill the New York Heart Association (NYHA) Class I-1Ile. Therefore, aerobic exercises should weeks to achieve physiological adaptation ${ }^{5}$ for the
patients with heart failures. Beneficial outcome results may be achieved when optimal physiological and psychosocial requirements are fulfilled
gerobic exercise varies from $15-60$ minutes. Auration of advised by physical therapist. It is advised initially that the patients with CHF should avoid physical activity and rest is results are obtained when exercise training is intromising among the patients. Recently, a moderate supervised exercise program for the patients who are in class NYHA class-II \& III are advised in order to improve the exercise still going on to clarify the type of exercises advised to the sill going on to clarify the type of exercises advised to the
patients with CHF and the effects of it.

\section{REFERENC}

[1] Lloyd-Williams E, Mair FS, Leitner, M. Exercise training and heart failure: a systematic review of current
evidence. The Br J Gen Pract 2002;52(474):47-55 Collins SP, Hinckley WR, Storrow AB. Critical review and recommendations for nesiritide use in the
emergency department. $J$ Emerg Med 2005;29:317-29,

[3] Kannel WB, Belanger AJ. Epidemiology of heart
failure. Am Heart t 1999:121.951-7.

[4] American Heart Association. Heart Disease and Stroke Statistics: 2005 Update. Dallas, Tex: AHA;2005. [5] Scottish Intercollegiate Guideline Network SIGN. Management of chronic heart failure: A national
clinical guidelines. 2007 [Online]. Available from: www.sign.ac.uk/pdf/sign95.pdf [Accessed ist March, 2011]

6] Khan Z, Khan B, Haider I, Khan I, ud- Din J, Khan $\mathrm{H}$ et al. Etiology of congestive heart failure at a tertiary

Ishaa M, Jamal Q. Treatment of Heart failure under

current guideline. Pak Heart J 2001;34(1-4):29-37

Piepoli MF, Conraads V, Corrà U, Dickstein K, Francis
DP, Jaarsma T et al. Exercise training in heart failure: the Heart Failure Association and the European Association for Cardiovascular Prevention and Pina IL, Apstein CS, Balady GJ, Bekardinelli R
Pind Chaitman BR, Duscha, BD et al. Exercise and hear Association committee on exercise, rehabilitation and prevention. Circulation 2003;107/8):1210-25

Exercise laylor RS, Singh S, Coats, A, Ebrahim s. Cochrane Database of Syst Rev 2004: (3). Mandic S, Riess K, Haykowsky MJ. Exercise training
for individuals with Coronary Artery Disease or Heart lor individuals with Coronary :
Failure. Physiother Can 2005:58:21-29

[12] Flynn KE, Piña LL, Whellan DJ, Lin L, Blumenthal JA, Ellis $S J$ e tal. Effects of exercise training on health status in patients with chronic heart failure:
HF-ACTION randomized controlled trial. JAMA 2009 Apr 8:301 (14):1451-8
Pozel B, Duncan

exercise training on fatigueg $M$. The effects of failure, Eur $J$ Cardiovas Nurs 2008 Jun; (2): : 127-32

14] ExTraMATCH Collaborative. Exercise training meta-analysis of trials in patients with chronic hear
failure (Cover story). BMJ 2004;328(7433):189-192.

[15] Davies EJ, Moxham T, Rees K, Singh S, Coats AJS,
Ebrahim $S$ et al. Exercise training for systolic heart Ebrahim $S$ et al. Exercise training for systolic heart
failure: Cochrane systematic review and meta-

analysis. Eur J Heart Fail 2010:12:706-715

Haykowsky MJ, Liang $Y$, Pechter $D$, Jones $L W$,
MC.Alister FA, Clark AM. A meta-analysis of the effect of exercise training on left ventricular remodon the type of training performed $1 \mathrm{Am}$ Conl Cardiol 2007;49(24):2329-2336. 117] Riegel B, Moser DK, Rayens MK, Carlson B, Pressler SJ,
Shively M et al. Ethnic Differences in Quality of Life in persons with heart failure. J Card Fail 2008; $14(1): 41-7$

18] Thow, MK (Ed). Exercise Leadership in Cardiac Rehabilitation-Evidence based Approach. 2006. Wiley and Sons, West Sussex.

[19] Magnusson G, Rong LK, Isberg B, Sylven C, Saltin B mportance of heart and skeletal muscle. Clinica Physiology 1996;16(2):183-195
[20] Witham MD, Argo IS, Johnston DW, Struthers $A D$. MCMurdo ME. Predictors of exercise capacity and every day activities in
Fail 2006:8(2);203-207

[21] Myers JN, Bubaker PH.Chronic Heat Folue Durstine 'JL, Moore GE (Eds.) ACSM's Exercise Management for Persons with chronic disease and disabilities 2nd Edition. USA: ACSM Group; 2003.

retamato Miyata $K$. Ito. K, et al Six-month aerobic exercises training ameliorates Central sleep apnea in patients

[23] Cao W. Shieh I Rana Ml, Haberl E Jones JD, Naughton Patients With Congestive Heart Failure Arch Phys Med Rehabii 2005:8(9):e 14

[24] Hambrecht R, Gielen E, Linke A, Fiehn E, Yu J, Walther $\mathrm{C}$, Schuler $\mathrm{N}$, Schuler $\mathrm{G}$. Effects of exercise resistance in patients with chronic heart failure: $\mathrm{A}$ randomized trial. JAMA 2000;283(23):3095-3101

[25] Sturm B, Quiltan $M$, Wiesinger GF, Stanek B, Frey B. Pacher R. Moderate-Intensity exercise training with
elements of step aerobics in patients with severe chronic heart failure. Arch Phys Med Rehabil 1999:80(7): $746-750$

[26] Dracup K, Evangelista LS, Hamiltion MA, Erickson V. Program on the Clinical outcomes in the heart failure. Am Heart J 2007:154(5):877-883

[27] Mezzani A, Corra U, Giannuzzi P. Central adaptations to exercise training in patients with

[28] Cowie A, Thow MK, Granat MH, Mitchell SL. A comparison of home and hospital-based exercise effects upon physical activity level. Eur J Prev Cardiol 2011 1;18(2) 158-166.

[29] Swank AM, Funk DC, Manire JT, Allard AL, Denny DM. Effect of resistance training and aerobic condi-
tioning on muscular strength and submaximal fitness Strength Cond Res age and gendio:24(5):1298-1305 